\title{
0 trabalho de fisioterapeutas de Núcleos de Apoio à Saúde da Família na assistência ao idoso
}

\author{
The work of Family Health Support Group Physical Therapists in elderly care \\ El trabajo de fisioterapeutas de Núcleos de Apoyo a la Salud de la Familia en la \\ asistencia al anciano
}

\section{Recebido: 13/09/2017 \\ Aprovado: 05/07/2018 \\ Publicado: 27/09/2018}

\author{
Ana Luíza Moreira Pauferro ${ }^{1}$ \\ Fabiane Ribeiro Ferreira ${ }^{2}$ \\ Paula Maria Machado Arantes ${ }^{3}$ \\ Rosana Ferreira Sampaio 4 \\ Júlia Baldoni ${ }^{5}$
}

Este estudo tem como objetivo investigar o processo de trabalho de fisioterapeutas do NASF de uma regional de saúde da cidade de Belo Horizonte. Trata-se de estudo descritivo, quantitativo, com dados de um questionário autoaplicado, respondido por 10 profissionais entre setembro e outubro de 2015. Os resultados demonstraram conformidade da maior parte das ações com as diretrizes relativas à atenção ao idoso e algumas inconformidades em relação às diretrizes do NASF, o que reforça a necessidade de ajustes na forma de atuação dos NASF, ainda em construção. Com ações que contribuem para redução de incapacidades, o fisioterapeuta atuando junto à equipe multiprofissional contribui para a assistência ao idoso na atenção primária.

Descritores: Atenção primária à saúde; Saúde da família; Assistência integral à saúde; Fisioterapeutas.

This study aims at investigating the work process of physical therapists from the NASF of a sanitary region of the city of Belo Horizonte. It is a descriptive and quantitative study, using data from a selfapplied questionnaire that was answered by 10 professionals from September to October, 2015. Results indicated that most actions were in accordance to the directives regarding elderly health care, although there were some actions that did not comply with NASF directives, which reiterates the need to adjust the way in which the actions of the NASF are carried out, as it is being created. Carrying out actions that contribute to diminish the disabilities, the physical therapist acts with the multiprofessional team and contributes for the assistance of the elder in the primary health care.

Descriptors: Primary health care, Family health; Comprehensive health care; Physical therapists.

Este estudio tiene como objetivo investigar el proceso de trabajo de fisioterapeutas del NASF de una regional de salud de la ciudad de Belo Horizonte. Se trata de un estudio descriptivo, cuantitativo, con datos de un cuestionario autoaplicado, respondido por 10 profesionales entre septiembre a octubre de 2015. Los resultados demostraron conformidad de la mayor parte de las acciones con las directrices relativas a la atención al anciano y algunas inconformidades en relación a las directrices del NASF, lo que refuerza la necesidad de ajustes en la forma de actuación de los NASF, aún en construcción. Con acciones que contribuyen a la reducción de incapacidades, el fisioterapeuta actuando junto al equipo multiprofesional contribuye a la asistencia al anciano en la atención primaria.

Descriptores: Atención primaria de salud; Salud de la família; Atención integral de salud; Fisioterapeutas.

\footnotetext{
1. Fisioterapeuta. Especialista em Geriatria e Gerontologia Belo Horizonte, Minas Gerais, Brasil. ORCID: 0000-0001-6386-0559 Email: ana.pauferro@gmail.com

2. Fisioterapeuta, Mestre em Ciências da Reabilitação. Doutora em Saúde Pública. Pós Doutora em Ciências da Reabilitação. Professora Adjunta da Universidade Federal de Minas Gerais (UFMG) Belo Horizonte, Minas Gerais, Brasil. ORCID: 0000-0003-3758-7493 E-mail: fabianerf@hotmail.com

3. Fisioterapeuta. Mestre, Doutora e Pós Doutora em Ciências da Reabilitação. Professora Adjunta da UFMG Belo Horizonte, Minas Gerais, Brasil. ORCID: 0000-0001-7064-9751 E-mail: paulamma@gmail.com

4. Fisioterapeuta. Especialista em Epidemiologia em Serviços de Saúde. Doutora em Saúde Pública. Pós Doutora em Saúde Coletiva. Professora Titular do Programa de Pós Graduação em Ciências da Reabilitação da UFMG Belo Horizonte, Minas Gerais, Brasil. ORCID: 0000-0002-4775-9650 E-mail: sampaioufmg@gmail.com

5. Fisioterapeuta. Especialista em Ergonomia. Mestre em Ciências da Reabilitação Belo Horizonte, Minas Gerais, Brasil. ORCID: 0000-0003-1587-1020 E-mail: juliabaldoni@gmail.com
} 


\section{INTRODUÇÃO}

Tuma perspectiva epidemiológica, o envelhecimento da população é acompanhado pelo incremento
relativo das doenças e condições crônicas de saúde, bem como de incapacidades associadas ${ }^{1}$. Assim, políticas públicas voltadas para a população idosa e para o novo perfil de saúde do país se fazem necessárias.

O Brasil vem desenvolvendo políticas públicas para este segmento. Em 2006, foi aprovada a Política Nacional de Saúde da Pessoa Idosa (PNSPI)2, que apresenta como finalidade a "recuperação, manutenção e promoção da autonomia e independência dos indivíduos idosos, por meio de medidas coletivas e individuais em todos os níveis de atenção".

No contexto do SUS, diante da necessidade de ampliação da abrangência das ações na Estratégia de Saúde da Família (ESF), foram criados, em 2008, os Núcleos de Apoio à Saúde da Família (NASF). Os NASF são equipes multiprofissionais, compostas por profissionais de diferentes áreas de conhecimento, que devem atuar de maneira integrada, sob a lógica de apoio matricial, com as Equipes de Saúde da Família (ESF) 3,4.

Em conformidade com o modelo de saúde orientado em redes de atenção, com a atenção básica como coordenadora do cuidado, a ESF deve representar, para o idoso, o principal vínculo com o sistema de saúde ${ }^{5}$. E, considerando que as políticas públicas para a população idosa têm a manutenção/recuperação da funcionalidade como foco da atenção integral, todas as ações de promoção da saúde, de assistência e reabilitação devem ter essa proposta como meta principal 2,6 .

Assim, os profissionais envolvidos na ESF devem apresentar competências específicas, de forma a garantir a realização das atividades pactuadas para a saúde da pessoa idosa ${ }^{2,6}$. Como parte integrante da atenção básica, o NASF também tem como missão o apoio, junto às ESF, ao cuidado integral à pessoa idosa, seguindo as mesmas diretrizes.

\section{A Política Nacional de Saúde da Pessoa Idosa}

A PNSPI foi resultado de uma necessidade de avançar nas práticas para a saúde integral da população idosa no SUS. Baseia-se no paradigma da funcionalidade para a conceituação de saúde da pessoa idosa, assumindo que a perda da capacidade funcional é o principal problema que pode afetar o idoso 2 .

Entre as diretrizes desta política estão: promoção do envelhecimento ativo e saudável; atenção integral e estímulo às ações intersetoriais, visando a integralidade; provimento de recursos capazes de assegurar qualidade da atenção; fortalecimento do controle social; formação e educação permanente dos profissionais de saúde do SUS; divulgação da PNSPI para profissionais de saúde, gestores e usuários do SUS².

\section{O NASF}

A atuação do NASF se baseia nas diretrizes relativas à atenção básica e se dá sob a forma de uma metodologia denominada apoio matricial, na qual profissionais de referência e especialistas mantêm relação entre si e compartilham campos de conhecimento de modo horizontal, de forma a gerar novos saberes e possibilidades de atuação, o que diminui a fragmentação do cuidado ${ }^{4,7}$.

A atuação dos profissionais do NASF deve abordar a promoção da saúde, a prevenção de agravos e a reabilitação, favorecendo o cuidado da população em todas as etapas da vida e fortalecendo a ESF. Devem ser priorizados os atendimentos compartilhados e intervenções aos usuários e famílias de forma articulada com as ESF. As formas mais comuns de assistência são a visita domiciliar, o atendimento individual e os grupos operativos. Uma importante atividade prevista no processo de trabalho do NASF é o projeto terapêutico singular (PTS).

\section{O profissional fisioterapeuta no NASF}

A inserção do fisioterapeuta no NASF representa a primeira aproximação formal da fisioterapia no nível da atenção básica. ${ }^{8}$ Desde sua origem, a fisioterapia tem um caráter essencialmente curativo e reabilitador. 
A atuação nas áreas de promoção e prevenção vem ocorrendo apenas recentemente ${ }^{8-10}$. Neste novo campo, há a necessidade de romper o isolamento e o individualismo da prática fisioterapêutica reabilitadora para emergir uma nova lógica de atuação em equipe multiprofissional e interdisciplinar, de forma a garantir integralidade na assistência 9 .

Em qualquer que seja o nível de atuação, a fisioterapia apresenta a funcionalidade como ênfase de seus objetivos terapêuticos, visando promover, ao máximo, o retorno do indivíduo à sociedade.

Nessa perspectiva, fica evidente a importância do fisioterapeuta para a atenção integral ao idoso no âmbito da ESF, pois seu foco de atuação vai ao encontro das diretrizes das políticas de atenção à saúde do idoso, que se direcionam ao enfrentamento das incapacidades ${ }^{10}$.

Diante do exposto, o objetivo deste estudo foi investigar o processo de trabalho de fisioterapeutas do NASF de uma regional de saúde da cidade de Belo Horizonte.

\section{MÉTODO}

Trata-se de um estudo observacional descritivo, com amostra composta por 10 fisioterapeutas que compunham o total de equipes de NASF de uma regional de Belo Horizonte $\mathrm{MG}$, no período de setembro a outubro de 2015.

Os profissionais foram convidados a participar do estudo por meio de contato pessoal, quando foram informados sobre os objetivos e procedimentos da pesquisa. De acordo com a conveniência do profissional, foi agendada uma visita ao local de trabalho, para explicação dos procedimentos da pesquisa e leitura do termo de consentimento livre e esclarecido.

Um questionário semiestruturado e autoaplicável foi adaptado para investigar as questões referentes à atuação dos fisioterapeutas $^{11}$. 0 instrumento constava de 89 questões abordando as seguintes dimensões: identificação geral, estrutura física e recursos, organização do apoio matricial às ESF, demanda específica da população idosa e gestão da demanda, educação permanente, produto final da assistência, satisfação profissional. A maior parte das questões era fechada, com a forma de resposta variando entre escalas de 0 a 10 , respostas simples e respostas múltiplas (mais de uma alternativa poderia ser assinalada).

Medidas de tendência central, dispersão e frequência foram utilizadas na análise dos dados, os quais foram processados no programa Microsoft Excel (2010). 0 estudo foi aprovado pelo Comitê de Ética e Pesquisa da UFMG, sob número de aprovação CEP 982.331.

\section{RESULTADOS}

Os resultados serão apresentados em itens utilizando-se quatro das sete dimensões do questionário, sendo: 1) Perfil dos participantes; 2) Estrutura física e recursos materiais; 3) Atividades previstas e apoio matricial; e 4) Demanda específica da população idosa e gestão da demanda.

\section{Perfil dos participantes}

Todos os 10 profissionais convidados a participar do estudo responderam ao questionário. Nove profissionais $(90 \%)$ eram do sexo feminino e um (10\%) do sexo masculino, com média de idade de 39 anos. Cinco $(50 \%)$ casados, quatro (40\%) solteiros e um $(10 \%)$ divorciado.

A carga horária semanal de todos os profissionais no NASF é de $20 \mathrm{~h}$ e $80 \%$ deles têm outro vínculo de trabalho. 0 tempo que trabalham no NASF variou de 2 anos e 11 meses a 8 anos, sendo que oito profissionais $(80 \%)$ trabalham há 6 anos ou mais. As equipes de NASF nas quais os entrevistados trabalham dão apoio a Equipes de Saúde da Família de mais de um Centro de Saúde. Cinco profissionais (50\%) dão apoio a três Centros de Saúde e outros cinco (50\%) a dois. Quanto ao número de Equipes que apoiam, quatro $(40 \%)$ relataram dar suporte a 8 equipes, um $(10 \%)$ a 9 , dois (20\%) a 11 e dois (20\%) a 12.

Todos os entrevistados completaram a graduação em instituições de ensino de Belo Horizonte - MG, com tempo de formação variando de 4 a 25 anos, sendo que $90 \%$ se formaram há 9 anos ou mais. Sete profissionais (70\%) possuem alguma formação no nível de pós-graduação, mas 
nenhuma delas na área de envelhecimento ou voltada à atenção básica.

\section{Estrutura física e recursos materiais}

Em relação aos espaços utilizados para o atendimento aos idosos (individual ou coletivo), o mais frequente foi o Consultório compartilhado com profissionais do NASF, seguido da Sala de Reunião/Grupos e de Espaços do território.

Quanto à adequação dos espaços para o atendimento da população em questão e para as atividades desenvolvidas (em uma escala de 0 a 10 , variando de muito ruim a excelente), a pontuação média foi 5,3 e 5,2, respectivamente. Em relação à suficiência de material e recursos para atendimentos individuais e coletivos, os profissionais, em sua totalidade, responderam negativamente.

No que se refere à disponibilidade de veículos de transporte para a realização de visitas domiciliares, $100 \%$ dos profissionais responderam positivamente, porém esta disponibilidade atende parcialmente à demanda do serviço.

\section{Atividades previstas e apoio matricial}

Os resultados referentes às atividades previstas e à forma como os profissionais disponibilizam suas agendas para as equipes de referência estão descritos nas tabelas 1 e 2 , respectivamente. Observa-se que as atividades realizadas são bem semelhantes entre as diferentes equipes de NASF, porém o mesmo não acontece na forma como a agenda é disponibilizada para as ESF.

Sobre a existência de protocolos e fluxos bem definidos, tanto de encaminhamentos para o NASF quanto para o planejamento de ações conjuntas NASF/ESF, a maior parte dos profissionais responderam negativamente, sendo $60 \%$ dos profissionais em relação à primeira questão e $100 \%$ em relação à segunda.

Tabela 1. Atividades previstas, segundo fisioterapeutas de uma Regional de Saúde. Belo Horizonte, 2015.

\begin{tabular}{lc}
\hline Atividades & $\mathbf{\%}^{*}$ \\
\hline Consultas individuais específicas para avaliação & 100,0 \\
Atendimentos individuais específicos para tratamento & 100,0 \\
Atendimentos compartilhados & 90,0 \\
Grupos terapêuticos e/ou grupos de educação em saúde & 90,0 \\
Visitas domiciliares & 100,0 \\
Reuniões de matriciamento com equipes de saúde da família & 100,0 \\
Ações compartilhadas com outras instâncias, como a assistência social ou & 60,0 \\
comunidade & \\
\hline mesmo respondente poderia responder a mais de uma questão. &
\end{tabular}

Tabela 2. Maneira de disponibilização da agenda para as equipes de referência, conforme fisioterapeutas de uma Regional de Saúde. Belo Horizonte, 2015.

\begin{tabular}{ll}
\hline Maneira de disponibilização da agenda & $\mathbf{\%}^{*}$ \\
\hline Verbalmente, durante as reuniões com as eSF & 40,0 \\
Escrita, durante as reuniões com as eSF & 10,0 \\
A agenda é afixada na(s) unidade(s) de referência em local visível & 60,0 \\
A agenda não é disponibilizada para as eSF & 30,0 \\
\hline mo respondente poderia responder a mais de uma questão.
\end{tabular}

No que se refere às reuniões com as ESF, todos os profissionais responderam que ocorrem mensalmente. A percepção da qualidade do matriciamento realizado nas reuniões (em uma escala de 0 a 10, variando de muito ruim a excelente) obteve média 5,3. Os principais fatores negativos que influenciam a qualidade do matriciamento apontados foram: falta de conhecimento das ESF sobre a proposta de matriciamento, falta de conhecimento das ações e a forma de atuação do NASF pelas ESF, falta de interesse e adesão das ESF às reuniões de matriciamento. 
Entre os principais fatores positivos percebidos sobre o matriciamento estão: "ampliação do olhar", "possibilidade de multiplicação e troca de conhecimentos entre os profissionais", "capacitação", "melhor indicação dos casos encaminhados", "maior resolubilidade dos casos" e "melhor direcionamento do usuário na rede". Em relação à elaboração de PTS, a maioria dos profissionais $(60 \%)$ responderam não realizar.

\section{Demanda específica da população idosa e gestão da demanda}

Sobre o número aproximado de encaminhamentos de pessoas acima de 60 anos recebidos mensalmente, dois (20\%) não souberam especificar, um (10\%) respondeu 7 e as demais (70\%) responderam 15 ou mais. Entre os 8 participantes que responderam, o número médio de encaminhamentos foi de 16,63 .

A principal motivação para encaminhamento de usuários com mais de 60 anos para o NASF pelas ESF é condição/diagnóstico clínico, conforme 70\% dos profissionais. Outros motivos são queixa do usuário ou da família e estado funcional do usuário.

Sobre os tipos de atendimentos que os fisioterapeutas realizam especificamente com o público idoso, a visita domiciliar e os grupos operativos foram os de maior proporção (Gráfico 1).

Gráfico 1. Atividades realizadas para a população idosa encaminhada, de acordo com fisioterapeutas de uma Regional de Saúde. Belo Horizonte, 2015.

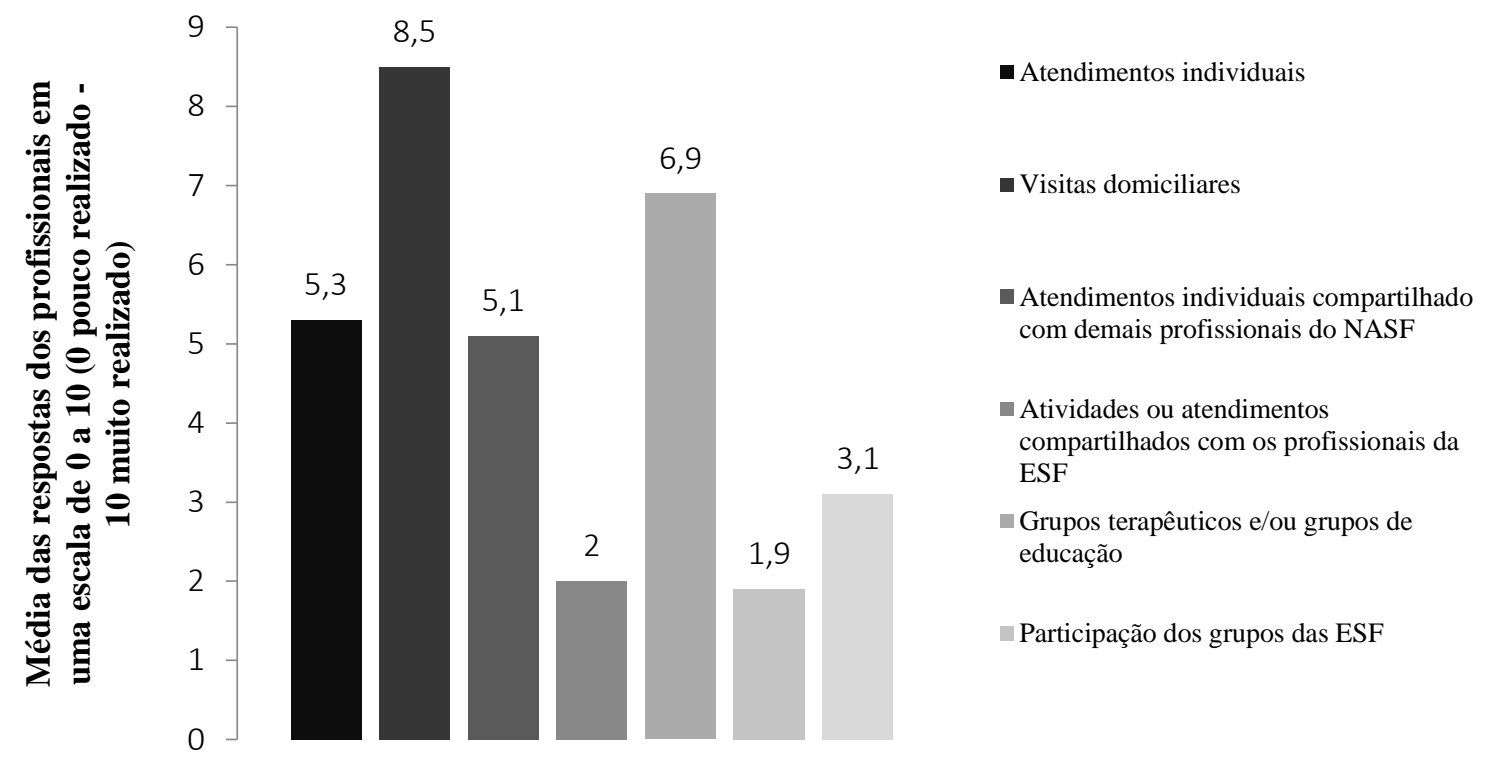

\begin{tabular}{lcrllll} 
As & condutas & \multicolumn{2}{c}{ frequentemente } & cuidador/familiar, & seguido & de \\
realizadas & com & esses & usuários: & encaminhamento para grupos estão no \\
acompanhamento & individual & com & Gráfico 2. & & & \\
orientações/treinamento & do & &
\end{tabular}


Gráfico 2. Condutas realizadas aos casos atendidos, conforme fisioterapeutas de uma Regional de Saúde. Belo Horizonte, 2015.

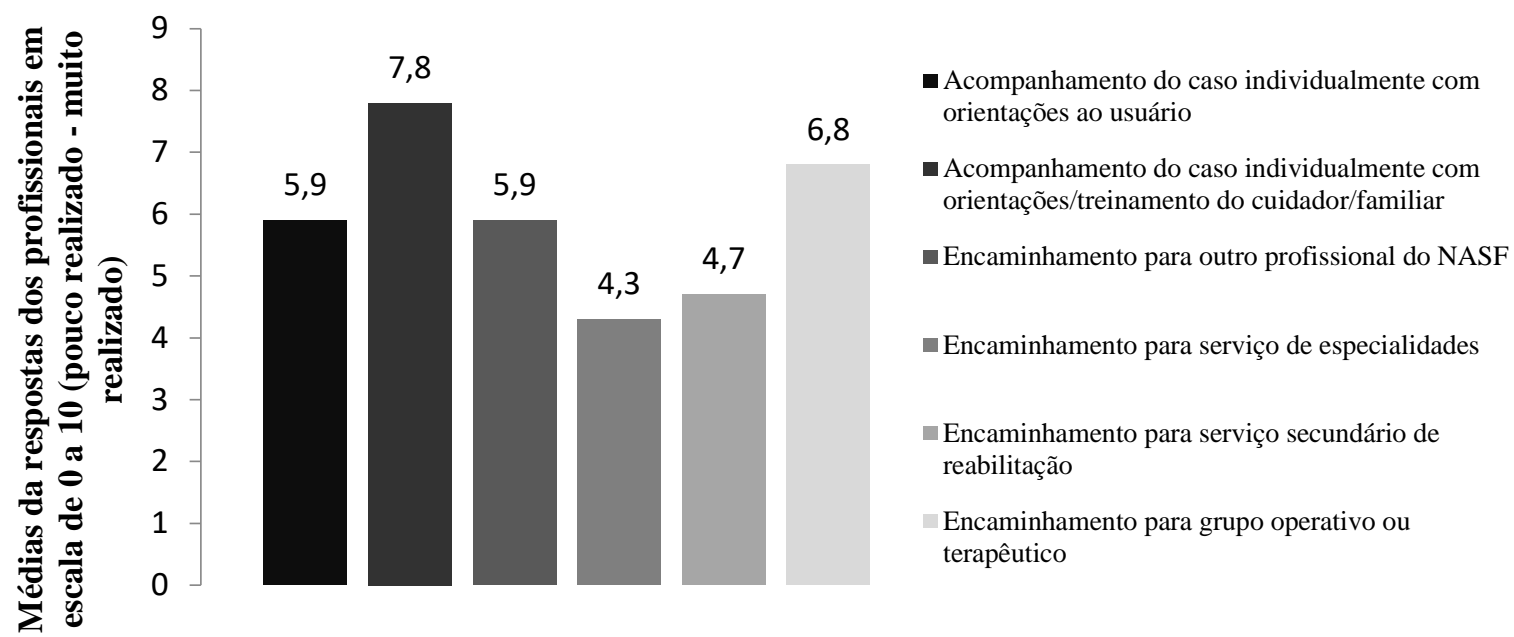

Entre as ações de promoção da saúde, prevenção de agravos e reabilitação, as ações de prevenção são as mais relatadas (83\%).

A média da percepção dos profissionais sobre a eficácia de suas ações para a manutenção/promoção/recuperação da funcionalidade do idoso, (em escala de 0 a 10 , variando de muito ruim a excelente), foi 6,44 .

Quanto à utilização do modelo da CIF como norteador da assistência aos usuários idosos, embora 70\% afirmem ter recebido formação específica sobre o assunto, apenas $40 \%$ afirmaram utilizar o modelo. No que tange à educação permanente, no último ano, somente um profissional (10\%) recebeu formação específica sobre temas relacionados ao envelhecimento.

Diante da conformação do sistema em RAS, cinco (50\%) afirmaram realizar contato frequente com instituições de outros níveis de atenção, e a mesma proporção de profissionais declarou nunca ter articulado ações com outras instâncias que não as da saúde.

\section{DISCUSSÃO}

A Diretriz dos NASF preconiza que eles atuem em parceria com os profissionais das ESF, compartilhando as práticas em saúde nos territórios sob sua responsabilidade 4 . Ao descrever a metodologia de trabalho de apoio

matricial e equipe de referência, um estudo ${ }^{7}$ enfatizou a necessidade de um rearranjo organizacional dos sistemas de saúde para que a retaguarda especializada não trabalhe de forma fragmentada, com mecanismos como o da referência e contra referência, e sim de maneira horizontal, na construção compartilhada de diretrizes clínicas e sanitárias.

Para isso, é necessário um funcionamento dialógico entre as equipes de referência, responsáveis por manter uma relação de coordenação longitudinal com os usuários adscritos e as equipes de apoiadores, responsáveis por contribuir com intervenções e/ou conhecimento especializado. ${ }^{7}$

Por ser um núcleo de apoio, as ações do NASF são indissociáveis e dependentes das ações das ESF12. Ainda assim, a baixa articulação entre essas duas equipes foi verificada no presente estudo pelo baixo índice de realização de atividades compartilhadas. A participação em seus grupos específicos e elaboração conjunta de projetos terapêuticos singulares, vão de encontro a esta diretriz básica.

Outro estudo ${ }^{11}$ sobre os indicadores de resultado dos NASF em Belo Horizonte também observou dificuldades dos profissionais do NASF em realizar ações conjuntas com as ESF. O contato entre referências e apoiadores deve ser direto e 
periódico e deve implicar sempre na construção de projetos terapêuticos integrados ${ }^{7}$.

Observa-se que, embora as reuniões conjuntas ocorram com frequência, os participantes percebem baixa qualidade do matriciamento dos casos. A essa baixa qualidade atribuem principalmente razões como o desconhecimento das ESF em relação à proposta de apoio matricial e às ações do NASF, desinteresse das ESF pelas reuniões de matriciamento e, ainda, escassez de informações relevantes sobre os usuários para auxiliar na condução dos casos.

Isso, possivelmente, é reflexo da dificuldade ainda existente em lidar com a nova forma de organização do trabalho e com ferramentas tecnológicas, como o apoio matricial e o projeto terapêutico singular ${ }^{11}$. Corroboram com esse achado, outros estudos sobre o NASF12-16, que ressaltam a necessidade de mudança na maneira de atuar dos profissionais envolvidos neste processo de cuidado.

Outra possível razão pode ser a baixa participação de algumas categorias profissionais da ESF nas reuniões e a grande rotatividade de profissionais, especialmente da categoria médica observada na prática ${ }^{11}$. Além disso, acredita-se que a inexistência de protocolos bem definidos de planejamento de ações conjuntas e de encaminhamentos para o NASF relatada pelos profissionais pode dificultar este diálogo essencial.

Esses achados revelam necessidade de ajustes relacionados ao processo de trabalho do NASF, de acordo com as diretrizes do Ministério da Saúde, o que ainda se mostra difícil, devido à necessidade de mudanças na lógica de atuação ${ }^{4}$.

0 perfil formativo da maior parte dos profissionais de saúde é considerado um obstáculo encontrado no percurso de implantação do NASF, uma vez que o trabalho em equipe não está presente em disciplinas curriculares da graduação ${ }^{15}$. Assim, uma revisão crítica dos processos de formação dos profissionais de saúde que favoreça a criação de oportunidades para a atuação conjunta, integrada e intersetorial se faz necessária frente à proposta de trabalho interdisciplinar do NASF13,15-17.

As atividades relatadas pelos participantes parecem contemplar a maior parte das ações previstas pela Política Nacional de Atenção Básica (PNAB) ${ }^{3}$. Similarmente ao que ocorre em relação às atividades realizadas especificamente ao público idoso, há uma assistência de adequada à heterogeneidade desta população, atendendo às diretrizes das políticas voltadas a ela.

A PNSPI ${ }^{2}$ valoriza 0 atendimento acolhedor e resolutivo, em ações que abrangem promoção, prevenção e tratamento de saúde, com prioridade para as atividades em grupo e que estimulem a convivência e a participação social. Portanto, o predomínio de realização de visitas domiciliares, seguidas de atividades coletivas encontrado é coerente com esta política e também com as diretrizes do NASF.

Mas, se por um lado as ações previstas estão sendo contempladas, por outro, a maior frequência de realização de atendimentos individuais do que de atendimentos compartilhados contradiz os princípios do apoio matricial para a priorização de atividades coletivas e/ou compartilhadas em relação ao atendimento direto e individualizado, que deve ocorrer somente em situações extremamente necessárias. ${ }^{7}$

Evidentemente, a forma de análise do presente estudo não permite afirmações assertivas acerca da diferença de frequência das ações. Contudo, fica claro que os profissionais percebem pouca realização de atividades compartilhadas com as ESF. Dados semelhantes foram encontrados em outro trabalho ${ }^{11}$ mostrando destaque para atendimentos individuais e tratamentos específicos, apesar da diversidade de ações realizadas pelos profissionais do NASF. Esse tipo de atendimento aumenta o risco de fragmentação do cuidado ${ }^{4,7}$. Isso confirma a dificuldade na efetivação do apoio matricial, bem como do uso de ferramentas como o PTS11.

Outros fatores dificultadores, como a falta de recursos materiais para realização de atividades (tanto individuais quanto 
coletivas), inadequação dos espaços físicos para as atividades desenvolvidas para a população idosa e disponibilidade insuficiente de transporte para atender a demanda para visitas certamente influenciam negativamente na realização de atividades compartilhadas. Nesse sentido, a fragilidade das unidades básicas é apontada pelo Ministério da Saúde como uma das dificuldades para a mudança das práticas na ESF em todo o Brasil ${ }^{11}$.

O predomínio de encaminhamento para grupos e acompanhamento com orientações em detrimento de encaminhamento para especialidades e nível secundário de reabilitação condiz com a PNAB e PNSPI, que preconizam que os usuários devem preferencialmente ser assistidos na atenção básica ${ }^{2,3}$, e, ainda, que $80 \%$ dos problemas de saúde devem ser solucionados neste nível de atenção ${ }^{18}$.

Porém, um importante fator a ser observado é que o NASF nunca deve assumir o papel de outros níveis de atenção ${ }^{13,14}$. Isso porque a insuficiência dos serviços especializados pode conduzir o NASF a um funcionamento equivocado, mas não é sua função abarcar a demanda de tratamentos individualizados não absorvidos pelas esferas responsáveis ${ }^{11,13,14}$. Especialmente no caso da reabilitação, que também apresenta acesso secundário reduzido. É relevante refletir sobre isso, pois o atendimento a tal demanda no nível primário pode acabar sufocando a possibilidade de desenvolvimento de outras atividades ${ }^{9}$.

Além disso é importante que as ações de reabilitação realizadas na atenção básica não se confundam com as realizadas em outros níveis de atenção. Para cumprimento do seu importante papel no contexto das RAS, uma das ações do NASF deve ser a de contribuir com a ESF para facilitar que usuários tenham acesso a rede de forma coerente e resolutiva, auxiliando na coordenação dos casos referenciados a outros serviços ${ }^{3}$.

Desta forma, o fato de apenas $50 \%$ dos entrevistados realizarem contato frequente com outros níveis de atenção está em desacordo com esta diretriz da atenção básica e da PNSPI, que preconiza a atenção integral e integrada à saúde do idoso, entre os níveis de atenção, incluindo a rede hospitalar e demais especialidades $^{2}$. Da mesma forma a pobre articulação intersetorial evidenciada pelos profissionais deste estudo também indica desacordo com a PNSPI no tocante as diretrizes do NASF, as quais estimulam ações intersetoriais e integração com outras políticas sociais como educação, esporte, cultura, trabalho, lazer e outras ${ }^{2,4}$.

Em relação à eficácia das ações dos fisioterapeutas do NASF para a manutenção / recuperação da funcionalidade dos idosos, observa-se que, de acordo com a percepção dos profissionais, estaria em torno de 64\%. Embora não tenham sido encontrados estudos com dado semelhante para comparação, levando-se em consideração a previsão potencial de resolução de $80 \%$ dos problemas na atenção básica ${ }^{18}$, esta média pode ser considerada razoável. Porém, visto que o paradigma de saúde para o indivíduo idoso se traduz pela sua condição de autonomia e independência ${ }^{2}$, acredita-se que este dado deveria ser mais discutido em outros estudos.

A percepção de distribuição equilibrada entre ações de promoção, prevenção de agravos e reabilitação pelos fisioterapeutas parece indicar que os mesmos estão sendo capazes de atuar de forma variada para atender as diferentes demandas que recebem. 0 predomínio de ações para prevenção de agravos é condizente com o nível de atenção em que estão atuando, podendo-se inclusive inferir que possivelmente os profissionais não estão sendo solicitados a realizar ações reabilitadoras próprias de outro nível de atenção. Além disso, realizar muitas ações de prevenção de agravos é condizente com a população em questão, pois é um grupo que sabidamente apresenta alta prevalência de doenças crônicas que podem afetar a funcionalidade.

Ainda na perspectiva da funcionalidade observou-se que a maior parte dos encaminhamentos pelas ESF é feita com base no diagnóstico clínico e não no diagnóstico funcional. Devido à complexidade 
inerente à saúde da pessoa idosa, o modelo de atenção centrado na doença não se mostra eficaz, sendo necessária uma abordagem global, multidimensional, que leve em conta a grande interação entre os fatores físicos, psicológicos e sociais, que devem ser abordados por uma equipe multiprofissional ${ }^{2}$.

Nesse sentido, a Organização Mundial de Saúde, em sua Classificação Internacional de Funcionalidade, Incapacidade e Saúde (CIF), considera a funcionalidade como uma interação dinâmica entre condições de saúde e fatores contextuais. Portanto, é um modelo biopsicossocial de atenção, tal como preconizado para atenção ao idoso, e possui conceitos muito úteis para a proposta metodológica de inter e transdisciplinaridade do apoio matricial ${ }^{2,18,19}$. Seu uso no SUS foi determinado em 2012, pelo Conselho Nacional de Saúde, entretanto, observou-se que apenas uma pequena parte dos participantes o utiliza em seu cotidiano de práticas, embora a maioria dos profissionais possua formação sobre o modelo.

Observa-se ainda que, embora algumas ações estejam em desacordo com diretrizes, em geral, o grupo deste estudo demonstrou conhecimento das diretrizes do NASF e aparentemente empenha-se em adequar sua atuação à metodologia proposta, embora encontre algumas barreiras.

0 fato de serem profissionais experientes na atuação no NASF (média de 6,2 anos) pode ser um facilitador. Reforça-se, no entanto, que do ponto de vista da atenção integral ao idoso, a melhora na articulação com as ESF e com a rede de serviços é necessária. Além disso, o modelo de atenção biopsicossocial deve ser utilizado para qualificar o diálogo entre profissionais e as decisões assistenciais ${ }^{19}$.

\section{CONCLUSÃO}

Após comparação do processo de trabalho dos fisioterapeutas com diretrizes do Ministério da Saúde (tanto em relação à diretrizes de trabalho do NASF como em relação às diretrizes da política que trata do cuidado ao idoso), observou-se que, de maneira geral, os profissionais apresentaram conformidade com as diretrizes relativas à atenção ao idoso, o que leva a acreditar que com suas ações variadas, que contribuem para redução de incapacidades, os fisioterapeutas, atuando junto à equipe multiprofissional contribuem de forma efetiva para a assistência ao idoso na atenção primária.

Já em relação às diretrizes do NASF, algumas inconformidades foram observadas, o que parece justificar a necessidade de ajustes na estruturação deste serviço enquanto política, bem como na formação profissional. Discussões, reflexões e atuações compartilhadas que rompam com a lógica tradicional e fragmentada da atenção são necessárias para abordagem da saúde dos usuários, tal como preconizado pelas políticas públicas e para ajustes na atuação dos núcleos, ainda em construção.

0 estudo teve como limitação a sua pequena amostra e o seu caráter apenas descritivo. Por outro lado, apresenta uma realidade que aponta o conhecimento e reconhecimento da realidade, na qual mais estudos sobre o processo de trabalho do NASF, em especial do fisioterapeuta sobre a assistência à pessoa idosa pelo NASF, são necessários.

\section{REFERÊNCIAS}

1. Mendes EV. As redes de atenção à saúde. Brasília, DF: Organização Pan-Americana da Saúde; 2011.

2. Ministério da Saúde (Br). Portaria ${ }^{\circ} 2528$, de 19 de outubro de 2006. Aprova a Política Nacional de Saúde da Pessoa Idosa [Internet]. Brasília, DF; 2006 [citado em 18 jul 2018]. Disponível em: http://bvsms.saude.gov.br/bvs/saudelegis/gm/2 006/prt2528_19_10_2006.html

3. Ministério da Saúde (Br). Política Nacional de Atençao Básica. Brasília, DF; 2012. v. 1, 110p.

4. Ministério da Saúde (Br). Departamento de Atenção Básica. Nucleo de Apoio à Saúde da Família: ferramentas para a gestão e para o trabalho cotidiano. Brasília, DF; 2014. v. 1, 118 p. (Cadernos de Atenção Básica; n. 39

5. Silvestre JA, Costa Neto MM. Abordagem do idoso em programas de saúde da família. Cad Saúde Publica. 2003; 19(3):839-47.

6. Veras R. Envelhecimento populacional contemporâneo: demandas, desafios e inovações Population aging today: demands, challenges and innovations. Rev Saúde Pública. 2009; 43(33):545-48. 
7. Campos GWS, Domitti AC. Apoio matricial e equipe de referência: uma metodologia para gestão do trabalho interdisciplinar em saúde. Cad Saúde Publica. 2007; 23(2):399-407.

8. Formiga NFB, Ribeiro KSQS. Inserção do fisioterapeuta na atenção básica: uma analogia entre experiências acadêmicas e a proposta dos núcleos de apoio à saúde da família (NASF). Rev Bras Ciênc Saúde. 2012; 16(2):113-22.

9. Bispo Júnior JP. Fisioterapia e saúde coletiva: desafios e novas responsabilidades profissionais. Ciênc Saúde Colet. 2010; 15(Suppl1):1627-36.

10. Aciole GG, Batista LH. Promoção da saúde e prevenção de incapacidades funcionais dos idosos na estratégia de saúde da família : a contribuição da fisioterapia. Saúde Debate. 2013; 37(96):9-10.

11. Araújo RE. Análise exploratória dos indicadores de resultados dos Núcleos de Apoio à Saúde da Família (NASF): a experiência de Belo Horizonte. [dissertação]. Belo Horizonte: Universidade Federal de Minas Gerais, UFMG; 2014. 121p.

12. Lancman S, Gonçalves RMA, Cordone NG, Barros JO. Estudo do trabalho e do trabalhar no núcleo de apoio à saúde da família. Rev Saúde Pública. 2013; 47(5):968-75.

13. Anjos KF, Meira SS, Ferraz CEO, Vilela ABA, Boery RNSO, Sena ELS. Perspectivas e desafios do núcleo de apoio à saúde da família quanto às práticas em saúde. Saúde Debate. 2013; 37(99):672-80.

14. Silva ATC, Aguiar ME, Winck K, Rodrigues KGW, Sato ME, Grisi SJFE, et al. Núcleos de apoio à saúde da família: desafios e potencialidades na visão dos profissionais da atenção primária do município de São Paulo, Brasil. Cad Saúde Pública. 2012; 28(11):2076-84.

15. Mângia EF, Lancman $S$. Núcleos de apoio à saúde da família: integralidade e trabalho em equipe multiprofissional [Editorial]. Rev Ter Ocup. 2008;19(2):1.

16. Macedo MAV, Ximenes-Guimarães JM, Coelho-Sampaio JJ, Pereira-Morais AP, Carneiro C. Análise do processo de trabalho no núcleo de apoio à saúde da família em município do nordeste brasileiro. Rev Gerenc Polít Salud. 2016; 15(30):194-211.

17. Souza TT, Calvo MCM, Resultados esperados dos Núcleos de Apoio à Saúde da Família: revisão de literatura. Saúde Soc. 2016. 25(4):976-87.

18. Campos RTO, Ferrer AL, Gama CAP, Campos GWS, Trapé TL, Dantas DV. Avaliação da qualidade do acesso na atenção primária de uma grande cidade brasileira na perspectiva dos usuarios. Saúde Debate. 2014; 38(Esp):252-64.

19. Alves MA, Ribeiro FF, Sampaio RF. Potencial de mudança nas práticas de saúde: a percepção de trabalhadores de uma rede de reabilitação em (trans) formação. Fisioter Pesqui. 2016; 23(2):185-92.

20. Quintana J, Ferreira E, Santos S, Pelzer M, Lopes M, Barros E. A utilização da Classificação Internacional de Funcionalidade, Incapacidade e Saúde no cuidado aos idosos. Referência. 2014; 4(1):145-52.

CONTRIBUIÇõES
Ana Luíza Moreira Pauferro e Fabiane
Ribeiro Ferreira participaram da concepção,
delineamento, análise e interpretação dos
dados, redação e revisão crítica. Paula Maria
Machado Arantes atuou na análise e
interpretação dos dados, redação e revisão
crítica. Rosana Ferreira Sampaio e Júlia
Baldoni contribuiu na redação e revisão crítica.

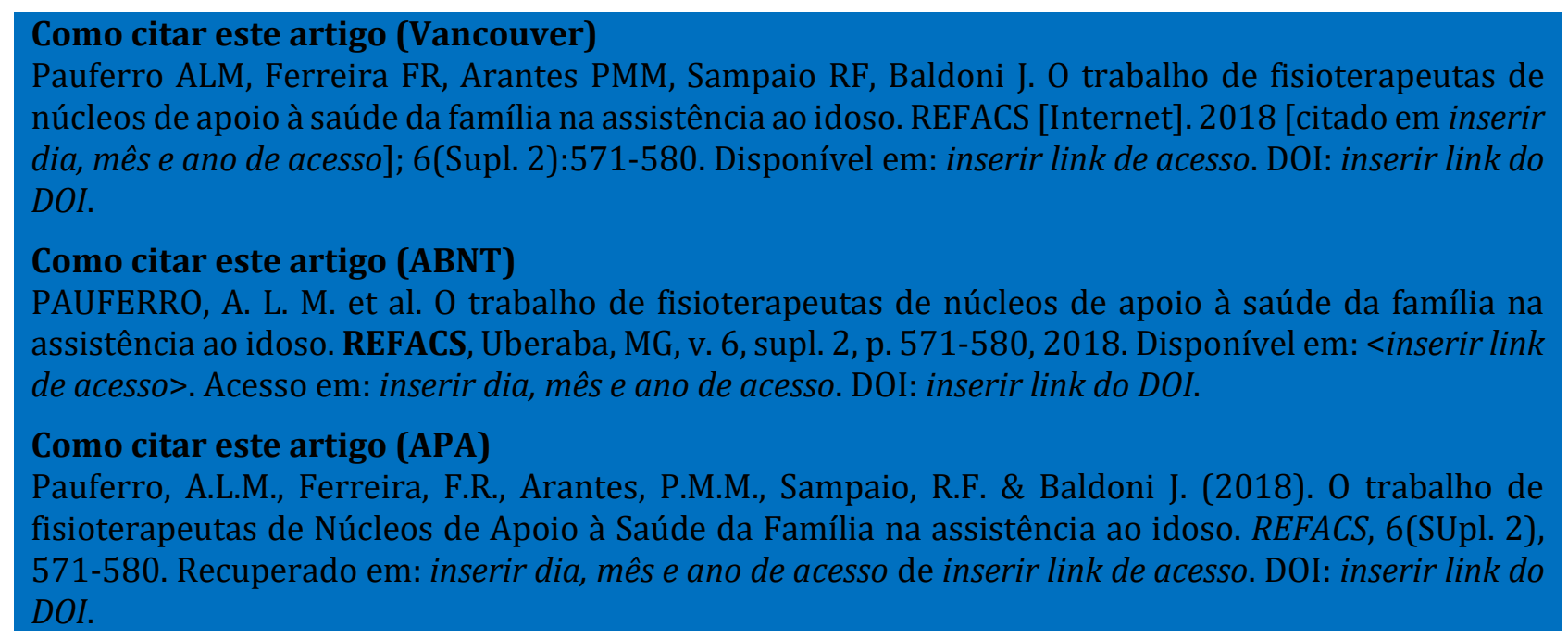

